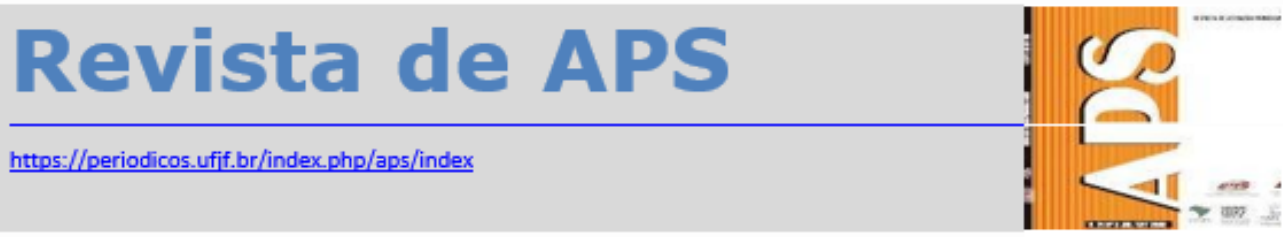

\title{
Rádio web na escola: um instrumento para prevenção contra o uso de drogas
}

\author{
Web radio at school: an instrument for drug use prevention
}

\author{
Deivson Wendell da Costa Lima, ${ }^{1}$ Deceles Ingrid de Carvalho Oliveira, ${ }^{2}$ Pâmella Costa \\ Queiroz, ${ }^{3}$ Tatiane Aparecida Queiroz, ${ }^{4}$ Ana Luiza de Oliveira Sousa ${ }^{5}$
}

\begin{abstract}
RESUMO
Este estudo teve como objetivo relatar a experiência da utilização da rádio web na escola como uma ferramenta para a prevenção contra o uso de drogas. Trata-se de um relato de experiência sobre o projeto de intervenção em uma escola estadual no interior do Rio Grande do Norte, desde agosto de 2016 até os dias atuais. Participaram a equipe de residentes multiprofissionais em Atenção Básica/Saúde da Família e Comunidade junto com a comunidade escolar, sob orientação e supervisão do Centro Regional de Referência para Formação em Políticas sobre Drogas. Promoveram-se oficinas sobre a estrutura de um programa de rádio, técnicas vocais, uso de redes sociais e mídias, exemplificação dos diferentes gêneros e formatos radiofônicos e construção dos roteiros. Nos programas, a temática das drogas foi abordada sob a ótica do fortalecimento de vínculos e de redução de danos, por meio de cordéis, textos, quiz, vinhetas e músicas. Na execução desse projeto, pode-se perceber o interesse e a autonomia dos alunos monitores em seu desenvolvimento, garantindo a obtenção de novas conquistas e
\end{abstract}

\footnotetext{
${ }^{1}$ Enfermeiro, doutorando pelo Programa de Pós-graduação em Enfermagem Psiquiátrica da Escola de Enfermagem de Ribeirão Preto da Universidade de São Paulo (EERP/USP). Docente da Faculdade de Enfermagem da Universidade do Estado do Rio Grande do Norte (UERN), em Mossoró (RN). E-mail: deivsonwendell@hotmail.com

${ }^{2}$ Atenção Básica/Saúde da Família e Comunidade da Universidade do Estado do Rio Grande do Norte (UERN) e Prefeitura Municipal de Mossoró (RN). Graduanda de Pedagogia pela UERN.

${ }^{3}$ Fisioterapeuta, especialista em Atenção Básica/Saúde da Família e Comunidade pelo Programa de Residência Multiprofissional em Atenção Básica/Saúde da Família e Comunidade da Universidade do Estado do Rio Grande do Norte (UERN) e Prefeitura Municipal de Mossoró.

${ }^{4}$ Enfermeira, especialista em Atenção Básica/Saúde da Família e Comunidade pelo Programa de Residência Multiprofissional em Atenção Básica/Saúde da Família e Comunidade da Universidade do Estado do Rio Grande do Norte e Prefeitura Municipal de Mossoró.

${ }^{5}$ Nutricionista, especialista em Atenção Básica/Saúde da Família e Comunidade pelo Programa de Residência Multiprofissional em Atenção Básica/Saúde da Família e Comunidade da Universidade do Estado do Rio Grande do Norte (UERN) e Prefeitura Municipal de Mossoró.
} 
aprendizados a cada programa realizado, fortalecendo vínculos na comunidade escolar e promovendo um crescente processo de corresponsabilização dos discentes com o próprio cuidado e aprendizado.

PALAVRAS-CHAVE: Serviços de saúde escolar. Prevenção primária. Transtornos relacionados ao uso de substâncias.

\section{ABSTRACT}

This study aimed to report the experience of using the web radio at school as a tool for the prevention of drug use. This is an experience report about the intervention project carried out at a state school in the countryside of Rio Grande do Norte state, from August 2016 to the present day. The Multiprofessional Residents team in Primary Care/Family and Community Health participated with the school community, under the guidance and supervision of the Regional Reference Center for Policy Training on Drugs. Workshops were organized on the structure of a radio program, vocal techniques, use of social media, on examples of different radio genres and formats and writing of scripts. In the programs, the drug thematic was approached from the viewpoint of the strengthening of bonds and damage reduction, through cordel literature, texts, quizzes, vignettes and songs. In the execution of this project, it was possible to perceive the interest and autonomy of the tutor students in their development, ensuring new achievements and knowledge in each program, strengthening bonds in the school community and promoting a growing process of co-responsibility of students with their own care and learning.

KEYWORDS School health services. Primary prevention. Substance-related disorders.

\section{INTRODUÇÃO}

A mídia, em suas diferentes formas de produzir comunicação, tem fundamental importância na propagação de orientações e informações de interesse coletivo e na geração de opiniões quanto à promoção da saúde que possibilitam a construção e o exercício de cidadania. ${ }^{1}$

Nesse sentido, o rádio é um veículo midiático de difusão de conhecimento e diálogo com as vivências de grupos sociais; é compreendido como um instrumento de educação das pessoas em se tratando de problemas sociais, entre eles o uso de drogas. ${ }^{2}$

A Agência de Notícias dos Direitos da Infância, em parceria com o Programa Nacional de DST/Aids do Ministério da Saúde ${ }^{3}$ e com especialistas da área de álcool e outras drogas organizaram um documento discutindo a questão da mídia e das drogas. Esse documento ressalta que a mídia exibe complexos problemas da sociedade, por exemplo a violência urbana, como sendo causados apenas pelas drogas, 
desconsiderando influências sociais, financeiras e políticas.

Contudo, faz-se necessária a difusão de debates mais aprofundados sobre a temática em espaços promotores de saúde para que, a partir de uma visão crítica, seja possível desmitificar discursos produzidos a respeito das drogas. ${ }^{4} \mathrm{~A}$ escola é considerada um importante espaço de discussão, reflexão e veiculação de informações sobre a prevenção ao uso de drogas. ${ }^{5}$

A utilização da ferramenta de rádio na escola é uma experiência inovadora que possibilita a construção do pensamento crítico entre os escolares por meio da disseminação de diferentes linguagens e produções radiofônicas. É relevante destacar que tal ferramenta contribui para o processo de educação entre pares, respeitando sua autonomia e produzindo conhecimentos em todas as fases do processo de elaboração dos roteiros, de edição, de execução e de avaliação dos programas de rádio na escola. ${ }^{6}$

A realização do projeto de intervenção da rádio web na escola surgiu da interlocução entre a Estratégia Saúde da Família (ESF) e a Universidade do Estado do Rio Grande do Norte (UERN). A equipe de residentes multiprofissionais em Atenção Básica/Saúde da Família e Comunidade, sob orientação e supervisão do Centro Regional de Referência para Formação em Políticas sobre Drogas (CRR), realizou aproximações com a realidade de uma escola de ensino médio inserida no território referente à Unidade Básica de Saúde (UBS).

A partir dessas vivências, as residentes identificaram adolescentes e jovens em condições socioeconômicas desfavoráveis decorrentes do uso de drogas lícitas e ilícitas, cujas famílias, em diversos casos, eram envolvidas com uso ou tráfico de drogas. Outra questão relevante observada pelas residentes foi à existência de uma estrutura de som e uma sala na escola com grande potencial para desenvolvimento da rádio como espaço de entretenimento e prevenção à saúde. Assim, o projeto de intervenção teve o intuito de atuar em um contexto que respondesse às necessidades dos adolescentes e jovens em vulnerabilidade social para redução de riscos associados ao uso de drogas, bem como possibilitassem o protagonismo juvenil, a educação entre pares e o envolvimento de toda a comunidade escolar.

Diante dessa realidade, este estudo teve como objetivo relatar a experiência da utilização da rádio web na escola como uma ferramenta para a prevenção do uso de drogas. 


\section{METODOLOGIA}

Este estudo é um relato de experiência do projeto de intervenção realizado pela equipe de residentes multiprofissionais em Atenção Básica/Saúde da Família e Comunidade da Universidade do Estado do Rio Grande do Norte (UERN), atuantes em uma UBS, como produto final do Curso de Formação para a Rede de Atenção a Usuários de Drogas promovidos pelo CRR.

A experiência iniciou em agosto de 2016, em uma escola estadual situada no interior do Rio Grande do Norte, tendo como público-alvo alunos de ensino médio com faixa etária entre 14 e 19 anos. A referida escola faz parte do território da UBS e é credenciada através do Programa de Saúde na Escola (PSE), sendo de responsabilidade da equipe de estratégia de saúde da família e residentes desenvolver ações de educação em saúde e promoção do cuidado. Dessa maneira, tem-se a pretensão de que o vínculo formado por meio do PSE e o espaço de discussões criado entre escola e UBS sejam utilizados de maneira longitudinal para o cuidado à saúde de adolescentes e jovens.

As atividades foram organizadas em momentos distintos desde a pactuação da equipe da UBS do bairro com a coordenação da escola, os professores e os alunos; até a realização dos programas de rádio tendo como tema central a prevenção ao uso de drogas lícitas e ilícitas.

\section{Momento: "Estreitando os Laços"}

Reunião entre a equipe da Unidade Básica de Saúde Duclécio Antônio de Medeiros e a equipe escolar composta por professores e alunos selecionados mediante participação no projeto da rádio escolar denominada Aida Mix. Na ocasião foram apresentados os objetivos da intervenção e acordadas datas para realização de cada uma das ações, bem como foram demonstrados os pontos-chave do projeto de intervenção, tendo como foco a criação de um espaço constante de discussões sobre a saúde dos adolescentes de maneira lúdica, utilizando os recursos disponíveis na escola para apresentar diversas temáticas, entre elas a prevenção do uso de drogas. 


\section{Momento: "Por Dentro do Rádio"}

Os alunos partícipes do projeto da rádio escolar, incluindo àqueles sensibilizados para participar do projeto de intervenção, foram convidados para uma oficina de rádio ministrada por um agente comunitário de saúde da UBS com formação em Comunicação Social. Este apresentou aos alunos a estrutura de um programa de rádio, as técnicas vocais utilizadas para melhorar a locução e o uso de redes sociais e mídias. Também realizou de maneira simplificada a apresentação e a exemplificação dos diferentes gêneros e formatos radiofônicos em que os alunos poderiam basear-se para estruturar os programas e melhor apresentar as temáticas propostas no projeto de prevenção ao uso de drogas. Utilizou-se como referencial os textos sobre gêneros radiofônicos ${ }^{7} \mathrm{e}$ termos técnicos. ${ }^{8}$

Os alunos foram também orientados quanto à organização da estrutura física da rádio, à estruturação do estúdio utilizando materiais recicláveis, ao uso de um programa de áudio como auxiliar na execução dos programas e à utilização de vinhetas.

Todos os conteúdos foram abordados de maneira clara e objetiva, utilizando-se exemplos para demonstrar os formatos radiofônicos, a diferença entre vinhetas e jingles e demonstrações de roteiros. Utilizou-se recurso audiovisual para auxiliar na explanação dos conteúdos.

\section{Momento: "Falando Sobre Drogas"}

Considerando a importância de se intensificar o diálogo sobre o uso de drogas, viu-se a necessidade de realizar encontros com os adolescentes e os jovens no intuito de serem facilitadores de informações as pessoas da mesma faixa etária na escola. Os alunos facilitadores receberam instruções para conduzir os programas de rádio e informações relacionadas à prevenção ao uso de drogas.

Inicialmente os alunos foram divididos em grupos para discutir temas como: o que é droga, motivações para o uso de drogas, tipos de drogas e seus efeitos, mitos e verdades sobre o uso de drogas e importância da escola na prevenção ao uso de drogas. Essas discussões ocorreram por meio da dinâmica "Fala Sério ou Com Certeza", em que foram apresentadas 11 afirmações referentes às temáticas e os alunos deveriam 
debater em pequenos grupos sobre a veracidade da informação e, em seguida, socializavam com o grande grupo. A dinâmica acontecia quando os alunos apresentavam a placa "Fala Sério" para indicar que o grupo julgou falsa a afirmação apresentada pelos facilitadores, e apresentavam a placa "Com Certeza" para indicar que o grupo julgava verdadeira a afirmação lançada. A mediação de cada grupo foi feita por uma das residentes da equipe multiprofissional da UBS Duclécio Antônio de Medeiros.

Em outro momento, os alunos, ainda em grupos, foram apresentados a algumas situações de conflitos relacionados ao uso de drogas. Pediu-se que expressassem opiniões de como abordar o adolescente e resolver os conflitos.

Todos os temas apresentados foram discutidos por meio de metodologias ativas, priorizando a participação dos jovens com suas linguagens menos técnicas e mais próximas da realidade em que estão inseridos, de modo que os facilitadores favoreceram a autonomia dos alunos, instigando a curiosidade e encorajando para a tomada de decisões e reflexão do problema apresentado. Utilizaram-se algumas metodologias descritas no fascículo "Álcool e outras drogas ", da coletânea Adolescentes e jovens para a educação entre pares, propostas pelo Ministério da Saúde. ${ }^{9}$

\section{Momento: "Mãos à Obra"}

Como produto final das oficinas de rádio sobre a prevenção contra o uso de drogas, os alunos facilitadores tiveram como encomenda a produção de programas de rádio que foram apresentados a toda a comunidade escolar em uma semana de cada mês, a qual foi intitulada "Semana de Prevenção ao Uso de Drogas".

Cada um dos grupos divididos no momento anterior pensou em gêneros radiofônicos que melhor se ajustavam aos temas trabalhados de forma a garantir o repasse adequado das informações à comunidade escolar.

Em outro momento oportuno, os alunos apresentaram as ideias à equipe de residentes multiprofissional e ao agente comunitário de saúde. Os alunos receberam orientações quanto à elaboração de roteiros de apresentação e à utilização de software instalado no computador da rádio para facilitar a transmissão de músicas e vinhetas.

Antes de os programas irem ao ar, realizou-se um breve ensaio com os locutores, sob orientação do agente comunitário de saúde, com o objetivo de realizar uma 
chamada à comunidade escolar, convidando-a a participar e interagir nas novas programações da Rádio Aida Mix.

\section{5o Momento: "Rádio Aida Mix e Prevenção ao Uso de Drogas"}

Durante os 15 minutos de intervalo os alunos apresentaram curiosidades sobre drogas, seus efeitos e os motivos que incentivam o consumo dessas substâncias. As temáticas foram abordadas por meio de cordéis, textos e quiz para a interação da comunidade escolar, além de vinhetas e músicas relacionadas ao tema.

Ao final de cada programa os alunos informaram, por meio de vinheta, os diversos serviços da Rede de Atenção Psicossocial ao Usuário de Drogas do município de Mossoró (RN).

\section{Momento: “Avaliando Nossas Ações”}

Ao final das transmissões dos programas de rádio, as residentes e o agente comunitário de saúde reuniram-se com os alunos facilitadores para avaliar cada uma das etapas da intervenção. Inicialmente pediu-se que cada aluno falasse um pouco da sua experiência nas atividades, em seguida foram entregues questionários com perguntas objetivas e subjetivas relacionadas às metodologias utilizadas, às potencialidades e às fragilidades identificadas pelos alunos e à importância de abordar a temática do uso de drogas na escola.

Atualmente o projeto de intervenção Rádio Aida Mix continua em execução com outras temáticas, entre elas: saúde mental e prevenção ao suicídio; sexualidade, prevenção de doenças sexualmente transmissíveis e uso de drogas; diversidade de gênero, respeito e uso de drogas; família e uso de drogas. Essas temáticas referem-se às demandas vivenciadas e identificadas pelos alunos na escola.

As atividades foram planejadas de acordo com o calendário escolar e a disponibilidade da equipe de residentes através de reuniões mensais com a equipe de alunos e professores da escola. Ademais, os residentes realizam capacitações voltadas para os alunos da escola, com uso de metodologias ativas, na perspectiva de ajudar no preparo do roteiro, da edição, da execução e da avaliação dos programas de rádio na 
escola.

\section{RESULTADOS E DISCUSSÃO}

A oficina de rádio web realizada pelo comunicador social teve grande repercussão entre todos os presentes. Nela, alunos e professoras envolvidos no projeto conheceram a sistematização dos programas de rádio, os modelos radiofônicos existentes, os tipos de transmissão de rádio e as técnicas para uma boa locução.

Outro estudo também retrata a oportunidade de inserção da mídia de rádio no contexto escolar. ${ }^{10}$ As oficinas promovidas apresentavam temas relacionados à vivência dos jovens e permeavam as discussões e o aprendizado das técnicas de rádio como: reportagem, notícia, spot, jingle, formatos e gêneros radiofônicos. Essas intervenções no ambiente escolar tiveram o rádio como instrumento tecnológico, permitindo a ampliação e a horizontalidade do diálogo, potencializando a construção de conhecimentos e da cidadania.

Nesse encontro, os participantes também foram capacitados para a utilização do software ZaraRádio, uma ferramenta gratuita de automação das emissões de rádio que proporciona agilidade na execução dos programas. ${ }^{11}$ Estudo aponta que a ferramenta é ideal para o desenvolvimento de uma rádio escolar, pois além de ser gratuita, oferece inúmeros recursos de manipulação. ${ }^{12} \mathrm{O}$ software é também utilizado pelos alunos da rádio escolar no município de São Miguel das Missões (RS), onde foi relatada a facilidade em manuseá-lo. ${ }^{13}$

A oficina transformou-se em um espaço de constante diálogo entre todos os participantes, de modo que os estudantes reconheceram a importância das discussões realizadas para o andamento do projeto.

No encontro denominado Falando sobre Drogas, no qual participaram alunos e professores envolvidos no projeto, foi possível observar que as metodologias utilizadas facilitaram a discussão da temática e permitiram o compartilhamento de vivências, opiniões e dúvidas a respeito do assunto. O caminho para a prevenção do consumo de drogas ocorre através da abertura de canais de comunicação e participação que promovam atividades alternativas, e não avaliativas, pela escola. Mais importante do que um discurso proibitivo é possibilitar espaços nos quais adolescentes e jovens 
possam vivenciar experiências significativas e compartilhá-las. ${ }^{14}$

A partir das discussões realizadas, observou-se que adolescentes e jovens percebem as drogas como algo prejudicial à convivência social e familiar, bem como para a saúde das pessoas que fazem uso, seja ele abusivo ou não. Reconhecem a influência dos fatores sociais e individuais para os usuários e discutem a importância do apoio social e familiar a eles, ressaltando que as medidas coercitivas e autoritárias na abordagem às pessoas em uso problemático não acarretam efeitos positivos; na verdade, impedem que eles busquem ajuda.

O uso de drogas é uma problemática complexa que traz impactos negativos à rede de relações do usuário, interferindo na maneira como ele vivencia o cotidiano. Instalada essa situação, os impactos passam a atingir também as relações sociais. Nesse sentido, são necessárias estratégias de apoio social que possam dar conta das demandas oriundas dessa condição. ${ }^{15}$

Os participantes destacam a importância da discussão dessa temática no âmbito escolar, sugerindo que deveria ser parte dos programas curriculares desde as séries iniciais do ensino fundamental, o que possibilitaria aos indivíduos reconhecer precocemente os problemas provocados pelo uso de drogas. No estudo realizado na cidade de Fortaleza (CE), ${ }^{5}$ os adolescentes também destacaram o papel da escola no desenvolvimento de uma visão crítica a respeito das drogas na prevenção de seu uso.

Os alunos relataram ainda que, no contexto familiar, existe um tabu em relação às drogas e que, por isso, os pais não falam nem orientam crianças e adolescentes sobre o assunto. O diálogo constitui-se um fator protetor ao uso de drogas, nesse sentido, é fundamental que a temática das drogas faça parte das orientações oferecidas pelos pais às crianças e aos adolescentes. ${ }^{16}$

Ao término das discussões, os alunos tiveram a oportunidade de colocar em prática os ensinamentos adquiridos, motivados a transformar a Rádio Aida Mix em um canal de informação e troca de experiências entre pessoas e grupos sobre o enfrentamento dos problemas de saúde, e não apenas em um espaço de entretenimento.

Para a produção dos programas de rádio na Semana de Prevenção ao Uso de Drogas foi utilizado o software básico ZaraRádio para transmissão dos conteúdos de áudio, como as músicas e as vinhetas informativas produzidas. Foram produzidos cinco 
programas para cada turno e abordados cinco temas referentes à prevenção ao uso de drogas. Os programas foram transmitidos ao vivo no horário do intervalo, com duração de aproximadamente 20 minutos. Ao final da programação os ouvintes foram orientados quanto à rede municipal de assistência aos usuários de drogas, além de informados sobre o fluxograma da Rede de Atenção Psicossocial ao Usuário de Drogas.

A produção dos programas ocorreu de maneira participativa e compartilhada entre alunos e professoras da escola promotora do projeto. As tarefas de elaboração de roteiros, escolhas das músicas, criação de gêneros textuais, locução dos programas e operacionalização do equipamento de som foram divididas entre os alunos de acordo com habilidades e afinidades de cada um, sob a orientação das professoras e residentes.

A interação entre alunos e professores e o estímulo ao protagonismo dos adolescentes também foram evidenciados no estudo "Práticas educomunicativas na rádio escolar: um estudo de caso na $A D I C-R^{\prime \prime}{ }^{17}$ que descreve a experiência de práticas educomunicativas em uma rádio escolar, ressaltando a importância da utilização da ferramenta de rádio na escola com a finalidade de aprimorar a comunicação entre professores e alunos, estimulando a produção coletiva e favorecendo a utilização de tais ferramentas educativas para o desenvolvimento do pensamento crítico.

Os alunos abordaram as temáticas do uso de drogas por meio de cordéis, textos informativos, apresentação de curiosidades, dados estatísticos e quiz com perguntas e respostas estimulando a participação da comunidade escolar. Também confeccionaram cartazes, relatórios e portfólios sobre cada um dos temas dos programas. A utilização de tais recursos em ações educativas de prevenção ao uso de drogas proporciona maior envolvimento da comunidade escolar, fortalecendo os alunos para decidir por melhor qualidade de vida e mudanças de hábitos e comportamentos. ${ }^{18,19}$

No estudo "A literatura de cordel como estratégia para promoção da saúde"20 foi demonstrada a viabilidade na utilização de cordéis como estratégia para promoção da saúde e prevenção ao uso de drogas, uma vez que a autora destaca a importância das ações de prevenção e estimulam a participação da sociedade e da família na multiplicação das ações preventivas, bem como trata-se de uma ferramenta de baixo custo.

Ao final dos programas educativos, alunos, professoras e residentes realizaram uma avaliação semelhante à experiência descrita em um relato de experiencia sobre 
educação popular acerca de DST via rádio comunitária, ${ }^{2}$ destacando pontos positivos e negativos. Os pontos positivos foram a iniciativa de confecção de outros materiais educativos de cada um dos temas apresentados durante as semanas de prevenção ao uso de drogas; o envolvimento de professoras nas ações; a interação da comunidade escolar para discussão das temáticas; e a abordagem da prevenção ao uso de drogas por meio da Rádio Aida Mix. Os pontos negativos foram a falta de organização de roteiros por parte de alguns alunos; e a carência na estrutura física da rádio, como a ausência das caixas de som e cabos de áudio. Identificou-se que, embora tenham ocorrido mudanças na dinâmica do intervalo, ainda foi possível observar pouco envolvimento da gestão escolar nas discussões propostas.

\section{CONSIDERAÇÕES FINAIS}

A utilização da mídia de rádio para o desenvolvimento do processo educativo foi uma estratégia inovadora de informação e divulgação sobre uso de drogas. Esse projeto de intervenção superou o modelo tradicional de educação em saúde, permitindo a construção coletiva de conhecimentos e a autonomia de adolescentes e jovens, de modo que estes, através de linguagem e ideias próprias trouxeram para a comunidade escolar informações fundamentais para a prevenção ao uso de drogas.

O projeto Rádio Aida Mix contribuiu com a formação de recursos humanos voltados para o funcionamento da mídia na escola como prevenção ao uso de drogas, e teve impacto no âmbito social em virtude de as atividades educativas serem realizadas pelos próprios alunos, o que favoreceu um diálogo com os pares e com os familiares, bem como trocas de experiências entre alunos e professores acerca do uso de drogas.

O projeto Rádio Aida Mix foi institucionalizado como atividade extracurricular e interdisciplinar pela gestão escolar que, junto com alunos, professores, profissionais da UBS e residentes assumiram a ferramenta como espaço de educação em saúde e de difusão de informações. Outras repercussões do projeto foram: recebimento de financiamento para custeio dos equipamentos e da infraestrutura acústica pela Secretaria Municipal de Educação; ampliação do número de monitores; e incorporação das atividades desenvolvidas pelos monitores como complementares no currículo. Ademais, o projeto foi apresentado em congressos nacionais nas áreas da saúde e da 
educação, e ainda em mídias televisivas locais.

A experiência foi fundamental no fortalecimento da articulação entre escola, Unidade Básica de Saúde e residentes multiprofissionais, pois as ações educativas ganharam destaque no ambiente escolar, deixando de ser algo pontual para ser um processo contínuo de construção de conhecimento. Observou-se o crescente interesse de alunos e professores no desenvolvimento do projeto, sendo garantida sua continuidade mensalmente, com a abordagem de diferentes temáticas em saúde sugeridas pela própria comunidade escolar.

\section{REFERÊNCIAS}

1. Janes MW, Marques MCC. The contribution of communication to health - a study on radio communication about risk in greater São Paulo. Saúde Soc. 2013 [cited 2018 Fev 12]; 22(4):1205-15. Available from: http://www.scielo.br/pdf/sausoc/v22n4/en 21.pdf.

2. Oliveira MLC. Vozes em sintonia: educação popular sobre DST via rádio comunitária. Interface (Botucatu, Online). 2014 [cited 2018 Fev 12]; 18(Supl 2): 1523-8. Available from: http://www.scielo.br/pdf/icse/v18s2/1807-5762-icse-18s2-1523.pdf.

3. Brasil. Ministério da Saúde (BR), Agência de Notícias dos Direitos da Infância, Programa Nacional de DST/AIDS. Mídia e drogas: o perfil do uso e do usuário na imprensa brasileira. Brasília: Ministério da Saúde; 2005. Available from: http://www.andi.org.br/file/50158/download?token=wnJ3ikoE.

4. Romanini M, Roso A. Mídia, ideologia e cocaína (crack): produzindo "refugo humano". Psico-USF. 2013 [cited 2018 Fev 12]; 18(3):373-82. Available from: http://www.scielo.br/pdf/pusf/v18n3/a04v18n3.pdf.

5. Costa AG, Camurça VV, Braga JM, Tatmatsu DIB. Drogas em áreas de risco: o que dizem os jovens. Physis. 2012 [cited 2018 Apr 08]; 22(2): 803-19. Available from: http://www.scielo.br/pdf/physis/v22n2/21.pdf.

6. Almeida LBC, Andrelo R. Rádio e TV escola: um projeto de capacitação para leitura e produção midiática na comunidade escolar. Rev Diálogos. 2011 [cited $2018 \mathrm{Apr}$ 08]; 15(1):26-33. Available from: https://portalrevistas.ucb.br/index.php/RDL/article/view/3546.

7. Barbosa Filho A. Gêneros radiofônicos. São Paulo: Paulinas; 2009. 158 p.

8. Mcleish R. Produção de rádio. São Paulo: Summus; 2001. 248 p.

9. Brasil. Ministério da Saúde. Secretaria de Vigilância em Saúde. Departamento de DST, Aids e Hepatites Virais. Adolescentes e jovens para a educação entre pares: álcool e outras drogas / Ministério da Saúde. Secretaria de Vigilância em Saúde. 
Departamento de DST, Aids e Hepatites Virais. Ministério da Educação. Secretaria de Educação Básica - Brasília: Ministério da Saúde; 2011. 56 p.

10. Tenorio LO, Shuvartz M. A rádio na escola: possibilidade interativa. Itinerarius Reflectionis. 2016 [cited 2018 Apr 08]; 12(1): 1-12. Available from: https://www.revistas.ufg.br/rir/article/view/37129.

11. Francisco DJ, Sobral SBDS. Rádio educação: a trajetória do programa rádio EDUCSE. Revista EDaPECI. 2010 [cited 2018 Apr 08]; 2(5): 72-90. Available from: https://seer.ufs.br/index.php/edapeci/article/view/588.

12. Porton SSAB. Prática edocomunicativa no espaço escolar: construindo ecossistemas comunicativos com a linguagem radiofônica [Dissertação]. Florianópolis: Universidade do Estado de Santa Catarina, Mestrado em Educação; 2015. $215 p$.

13. Stochero AD, Nilson LL. Perspectivas da implantação de uma rádio escolar: uso de equipamentos tecnológicos e o desenvolvimento de habilidades. Encontro Anual de Tecnologia da Informação e Semana Acadêmica de Tecnologia da Informação; 2015; Frederico Westphalen; EATI. p. 95-101.

14. Rodrigues EB, Abaid JLW. Prevenção do uso de drogas no âmbito escolar: uma revisão sistemática. Disciplinarum Scientia. 2013 [cited 2018 Apr 08]; 14(2): 17390. Available from: https://www.periodicos.unifra.br/index.php/disciplinarumCH/article/view/1756.

15. Pandini A, D'artibale EF, Paiano M, Marcon SS. Rede de apoio social e família: convivendo com um familiar usuário de drogas. Cienc Cuid Saúde. 2016 [cited 2018 Apr 08]; 15(4):716-22. Available from: http://www.periodicos.uem.br/ojs/index.php/CiencCuidSaude/article/view/34602

16. Belotti M, Fraga HL, Belotti L. Família e atenção psicossocial: o cuidado à pessoa que faz uso abusivo de álcool e outras drogas. Cad Bras Ter Ocup. 2017 [cited 2018 Apr 08]; 25(3):617-25. Available from: http://www.cadernosdeterapiaocupacional.ufscar.br/index.php/cadernos/article/ view/1727.

17. Silva EV, Gomes AL. Práticas educomunicativas na rádio escolar: um estudo de caso na ADIC-RN. ARTEFACTUM - Revista de Estudos em Linguagens e Tecnologia. 2016 [cited 2018 Apr 08]; 13(2): 1-12. Available from: http://artefactum.rafrom.com.br/index.php/artefactum/article/view/1344.

18. Carnevali MCC. Uso da mídia de rádio e da mídia escrita como objeto de prevenção ao uso de drogas lícitas e ilícitas [Trabalho de Conclusão de Curso]. Porto Alegre (RS): Centro Interdisciplinar de Novas Tecnologias na Educação Universidade Federal do Rio Grande do Sul; 2013. 57 p.

19. Silva JH et al. A educação em saúde na prevenção ao uso de drogas. Rev Conexão UEPG. 2014 [cited 2018 Apr 08]; 10(2):182-9. Available from: http://www.revistas2.uepg.br/index.php/conexao/article/view/6741/4279.

20. Lopes IC. A literatura de cordel como estratégia para promoção da saúde. Rev Enferm UFPE on line. 2015 [cited 2018 Apr 08]; 9(6): 8627-35. Available from: 
http://dspace.bc.uepb.edu.br/jspui/bitstream/123456789/9468/1/PDF\%20\%20lzabel\%20Cristina\%20Paulo\%20Silva\%20Lopes.pdf.

Submissão: maio de 2018.

Aprovação: outubro de 2018. 